\title{
Factores determinantes de la insolvencia empresarial: caso aplicado a la Bolsa Mexicana de Valores
}

\author{
Determinants factors of business insolvency: \\ the case applied to the Bolsa Mexicana de Valores \\ Ángel Virgilio Estrada Berlanga, Eduardo Javier Treviño Saldivar y Klender Aimer Cortez \\ Alejandro \\ Universidad Autónoma de Nuevo León
}

\begin{abstract}
The objective of this research it to contribute to the knowledge of the impact financial and economic factors on the business insolvency of public companies listed on the stock exchange. In addition, a comparative analysis is carried out between companies and sectors that incurred insolvency and those that have not to do this, the methodology is used with panel data. The information in this study comes from public companies that have listed on the Mexican stock exchange in the last 26 years. The results indicate that financial, non-financial and macroeconomic factors are the determinants in business insolvency; on the other hand, in the multisectoral model, the sectors most likely in fall into business insolvency are the frequent consumer products sector, the industrial sector, followed by the non-basic consumer goods and services sector, the one with the less risk is the material sector.
\end{abstract}

Keywords: business insolvency, models with panel data, prediction, public enterprises, sector studies.

JEL: G33, C23, C53, L32, L60.

\section{Resumen}

El objetivo de la presente investigación es contribuir al conocimiento sobre el impacto que tienen los factores financieros y económicos en la insolvencia

Factores determinantes de la insolvencia empresarial: caso aplicado a la Bolsa Mexicana de Valores. 
empresarial de las empresas públicas que cotizan en la Bolsa Mexicana de Valores (BMV) y se realiza un análisis comparativo entre empresas y sectores que han incurrido en insolvencia y las que no. Para ello se utiliza la metodología de probit con datos panel. La información del presente estudio proviene de empresas públicas que han cotizado en la Bolsa Mexicana de Valores en los últimos 26 años. Los resultados indican que los factores financieros, no financieros y macroeconómicos son las determinantes en la insolvencia empresarial y por otra parte en el modelo multisectorial los sectores que tienen más posibilidad de caer en la insolvencia empresarial son el sector de productos de consumo frecuente, el sector industrial, seguido por el sector de servicios y bienes de consumo no básico ya que el que menor riesgo tiene es el sector de materiales.

Palabras clave: insolvencia empresarial, modelos con datos de panel, predicción, empresas públicas, estudios sectoriales.

\section{Introducción}

En los últimos dos siglos el crecimiento económico a nivel mundial ha ido en aumento. Esto regularmente viene acompañado de bienestar y crecimiento en la calidad de vida. Un mayor desarrollo económico genera nuevos desafíos para la economía global. Las previsiones para el crecimiento económico han ido a la baja desde el comienzo del año 2019. En la mayoría de las regiones como América Latina, Medio Oriente y África del Norte se esperaba que el crecimiento económico aumentara y no fue así, incluso fue menor que el del año 2018 (BM, 2019).

Esta debilidad económica ha afectado a las principales empresas que propició la desaparición de éstas. Las continuas fricciones comerciales y en particular la disputa entre Washington y Beijing, el preocupante riesgo del desorden causado de Brexit del Reino Unido, el impacto del pronunciado gasto industrial en Estados Unidos y la desaceleración del consumo que ha tenido China han sido algunas de las causas de que grandes empresas sigan desapareciendo a nivel mundial (Kuger, 2019).

En el caso de México el panorama para las empresas no es muy diferente al del resto del mundo. Según el INEGI (2016) 33\% de las empresas mueren en su primer año y de las que continúan superviviendo $65 \%$ desaparecen a los cinco años. Aquellas que llegan a los 10 años de vida $76 \%$ desaparecen y sólo 18\% de las que llegan a los 15 años superviven.

\section{Estrada Berlanga et al.}


De esta manera entre más tiempo estén en operación tienen mayor probabilidad de insolvencia empresarial (IE). Y de los negocios que cuentan con 20 años de vida $86 \%$ desaparecen y sólo $11 \%$ de los negocios se mantienen operando en sus primeros 25 años. De igual manera en las empresas públicas existe un decremento en la participación dado que en 1993 un total de 190 empresas públicas estaban registradas y para el año 2019 solamente existían 145 dentro de la Bolsa Mexicana de Valores (BMV).

Entre las razones para que las empresas desaparezcan se encuentran aspectos de liderazgo, de gestión administrativa, del entorno macroeconómico y del sector al que pertenecen (Altman et al., 2019). Una de las principales consecuencias de la insolvencia empresarial es el desempleo. Éste deteriora el bienestar socioeconómico en las familias y en la sociedad (Pinzón, 2017; García et al., 2009; Cabrera et al., 2006).

Los estudios sobre el tema de insolvencia empresarial inician con los trabajos de Fitzpatrick (1932) en donde básicamente analizaba la tendencia y el comportamiento de ciertas razones financieras. La teorización de esta idea fue a partir de los trabajos de Beaver y Altman ya que estos autores crearon dos tipos de modelos. El primero de ellos propuesto por Beaver (1966) bajo un modelo univariable demostró tener una precisión del $87 \%$ en la predicción de la insolvencia utilizando razones financieras de liquidez, solvencia y rentabilidad. El segundo de ellos propuesto por Altman (1968) considera un modelo multivariable pionero en la predicción de insolvencia empresarial conocido como modelo Z-score.

A pesar de la gran aceptación del modelo de Altman en los años 80 aún se tenía ausencia de normalidad en las variables independientes, razón que surgen los trabajos de Ohlson (1980) y el de Zmijewski (1984) que utilizan métodos de probabilidad con funciones tipo logit y probit respectivamente. Estos modelos se centraron en mejorar el método estadístico de Altman y proponen un modelo que busca predecir la probabilidad de fracaso más que asegurar el fracaso como un evento histórico. A principios de los años 90 surgen los modelos de redes neuronales, siendo muy populares hasta el día de hoy. Con este método se pueden sintetizar algoritmos mediante un proceso de aprendizaje en donde los académicos consideran que no es necesario conocer los detalles técnicos y la solución de problemas no lineales (Rahimian et al.,1993).

Factores determinantes de la insolvencia empresarial: caso aplicado a la Bolsa Mexicana de Valores. 
La literatura de la insolvencia empresarial actualmente se encuentra orientada hacia países desarrollados, además no se aborda de manera multisectorial, se realiza con periodos de tiempos limitados y sin considerar factores externos e internos simultáneamente (Altman et al., 1995; Ibarra, 2002).

El objetivo de la presente investigación es contribuir al conocimiento sobre el impacto que tienen los factores financieros y económicos que generan la insolvencia empresarial en las empresas públicas que cotizan en la BMV. Además, se busca analizar los sectores más afectados por esta situación. Para esto se utiliza el método de probit y basándose en el modelo de Zmijewski (1984), dicho método econométrico se aplica con datos panel. La información de este estudio proviene de empresas públicas que han cotizado en la Bolsa Mexicana de Valores en los últimos 26 años.

La contribución de esta investigación es analizar el problema de la insolvencia empresarial considerando simultáneamente aspectos financieros, no financieros, macroeconómicos y con un enfoque multisectorial con el fin de que las empresas tomen decisiones con anticipación para evitar el desempleo y redireccionar el rumbo de su empresa. Con esto buscamos aumentar la probabilidad de alcanzar el éxito empresarial y además aportar al desarrollo de la teoría de la insolvencia empresarial.

Los resultados de esta investigación demuestran que las razones financieras de rentabilidad, liquidez, endeudamiento y flujo de efectivo operativo, además los factores no financieros de tamaño, crecimiento, edad y finalmente los factores macroeconómicos como tipo de cambio neto, riesgo país y riesgo de mercado son las variables más significativas que impactan en la insolvencia empresarial. En el modelo multisectorial los sectores que tienen mayor probabilidad de caer en la insolvencia empresarial son el sector de productos de consumo frecuente, el sector industrial, seguido por el sector de servicios y bienes de consumo no básico, el que menor riesgo se tiene es en el sector de materiales.

El artículo se divide en cuatro secciones: en la primera sección se hace la revisión de la literatura donde se abordan las principales variables de la insolvencia empresarial, en la sección dos se presenta el método utilizado para analizar el efecto que tienen los factores financieros, no financiero y macroeconómicos sobre la probabilidad de caer en insolvencia empresarial, en la sección tres se presentan los resultados obtenidos y finalmente en la

\section{Estrada Berlanga et al.}


sección cuatro presentamos las conclusiones en donde se discuten los principales hallazgos.

\section{Marco teórico}

Actualmente no existe una teoría de la insolvencia empresarial aunque son varias las teorías que surgen de la administración que fundamentan el tema (Ruiz, 2010). La teoría de la toma de decisiones y la teoría evolucionista de la empresa explican el efecto que tienen los factores internos de la empresa sobre su insolvencia y por su parte la teoría de sistemas y la teoría de la organización industrial relacionan el impacto de los factores externos sobre la insolvencia empresarial.

La teoría de la toma de decisiones explica el comportamiento humano considerando a la empresa como eje central para la toma de decisiones (Simón,1962). El tema de la insolvencia empresarial es tan sólo un eslabón dentro de la cadena de decisiones (Forrester, 1968). La teoría evolucionista considera como eje fundamental el conocimiento en beneficio de la empresa (Winter, 1996). Con base en estas teorías para tener el conocimiento necesario sobre la rentabilidad, liquidez, endeudamiento y flujo de efectivo operativo de una empresa facilita la toma de decisiones en favor de ésta. De esta manera y entre algunos de los factores internos de la empresa que determinan la insolvencia empresarial se encuentran las razones financieras y las variables de control. Las razones financieras son la de rentabilidad, liquidez, endeudamiento y flujo de efectivo operativo (Almamy et al., 2016; Arroyave, 2018; Beaver et al., 2005; Darrat et al., 2016; Espinosa et al., 2015; Tinoco y Wilson, 2013). Las variables de control son la edad de la empresa, su tamaño y el crecimiento de sus ventas (Abidali y Harris, 1995; Hsu y Wu, 2014; Platt y Platt, 2012; Madrid-Guijarro et al., 2011).

Con relación en la rentabilidad ésta tiene dos conceptos: la rentabilidad económica y la rentabilidad financiera. La rentabilidad económica se refiere a la mayor o menor capacidad que tienen las empresas para gestionar las inversiones y por otro lado existe la rentabilidad financiera y se refiere a la capacidad que tiene la misma empresa para generar riqueza hacia sus propietarios y depende directamente del multiplicativo causado por la rentabilidad económica y el grado de endeudamiento 0 apalancamiento (Segura, 1994). La rentabilidad como razón financiera es una métrica de gran popularidad en la literatura financiera, además es utilizada en modelos precursores de predicción de la insolvencia como en el modelo propuesto por

Factores determinantes de la insolvencia empresarial: caso aplicado a la Bolsa Mexicana de Valores. 
Fitzpatrick (1932) después en el modelo de Beaver (1966), Altman (1968), Ohlson (1980) y hasta la fecha en los modelos de redes neuronales como el de Kim et al. (2018). Los hallazgos de estos estudios señalan que existe una relación inversa entre la rentabilidad financiera y la insolvencia empresarial.

El concepto de liquidez se refiere a cumplir con los compromisos a corto plazo y a la destreza para convertir en efectivo determinados activos y pasivos (Freire et al., 2016). La liquidez también aparece en la gran mayoría de la literatura en los modelos de predicción de insolvencia (Altman et al., 2019). Winakor y Smith (1935) y Beaver (1968) encontraron un impacto significativo de la liquidez sobre la insolvencia empresarial. En la mayoría de la literatura financiera esta razón financiera aparece en todas las etapas de los estudios de insolvencia empresarial con un impacto negativo (Altman y Suggitt, 2000), esto es un aumento de la liquidez se relaciona de manera inversa con la insolvencia debido a que la falta de ésta conlleva al incumplimiento de compromisos adquiridos por la empresa.

El endeudamiento, según Brealey et al. (2015), también conocido como la estructura de capital, hace referencia a la forma en que los activos están financiados, los recursos privados son básicamente obtenidos de instituciones privadas y los recursos públicos son aquellos que se adquieren del mercado de capitales como son bonos y pagarés. El endeudamiento como razón financiera se empezó a utilizar en los modelos de predicción de insolvencia con Altman (1968). Otros estudios han encontrado que a mayor endeudamiento de la organización mayor es el riesgo de caer en insolvencia empresarial (Bathory, 1984; Ibarra, 2002; Montalván et al., 2011; Ohlson, 1980). Entre mayor endeudamiento tenga la empresa, ésta tendrá menos posibilidades de aprovechar las oportunidades que se presenten y se vuelve más vulnerable a choques en el mercado debido a que su capital lo tiene comprometido.

Sobre el concepto del flujo de efectivo, según Uhrig-Homburg (2005), éste se define como la variación de entradas y salidas de dinero en un periodo determinado. Bukovinsky (1994) utilizó flujo de efectivo en su estudio de insolvencia empresarial y lo separó en dos partes, la parte de la utilización del flujo de efectivo operativo para el cumplimiento de los pagos a corto plazo y los cambios que tuvo el flujo de efectivo neto dentro de las actividades restantes. El flujo de efectivo operativo mejoró la significancia del resto de las razones financieras a estudiar (Almamy et al., 2016; Aziz y Lawson, 1984;

\section{Estrada Berlanga et al.}


Casey y Bartczak, 1985; Gentry et al., 1985; Gombola et al., 1987). Cuando el flujo de efectivo operativo es mayor en una empresa es un indicador de un buen desempeño debido a que genera liquidez para hacer frente a los compromisos que se tienen a corto plazo.

Las variables internas de control mejoran el desempeño de los modelos de predicción ya que éstas se refieren a la edad, crecimiento y tamaño de la empresa (Madrid-Guijarro et al., 2011; Pervan y Kuvek, 2013). Abidali y Harris (1995) demostraron que una combinación de las razones financieras con las variables de control como la edad, tamaño y crecimiento explican de mejor manera el impacto que tienen estas en la insolvencia empresarial.

En cuanto a los factores externos las teorías que impactan en la insolvencia empresarial son la teoría de sistemas y la teoría de la organización industrial. La teoría de sistemas analiza a la organización y su interacción con el entorno. De esta relación resulta el desarrollo empresarial (Katz y Kanh, 1978). La teoría de la organización industrial estudia y analiza la estructura de los mercados y las formas de interacción de las empresas (Schmalensee, 1985). Estas teorías resaltan la importancia de los factores externos en el desempeño de las empresas y la manera en que estos se relacionan con los factores internos que influyen en la insolvencia empresarial (Foster, 1986). Según Rees (1990) indica que el desempeño de una empresa no sólo depende del liderazgo y la gestión administrativa sino que hay factores externos que influyen en ella. Altman et al. (1995) demostraron que el modelo Z-score estaba diseñado para países desarrollados que presentan una alta estabilidad macroeconómica y no era funcional para economías emergentes caracterizadas por una alta volatilidad y considerar los aspectos macroeconómicos permitió identificar la relación entre los factores internos y la insolvencia empresarial.

Entre los factores externos en la literatura que tienen mayor influencia sobre la insolvencia se encuentran el riesgo de país, el tipo de cambio, el riesgo de mercado y las recesiones económicas (Agarwal y Taffler, 2008; Campbell et al., 2011; Christidis y Gregory, 2010; Dambolena y Khoury, 1980; Hillegeist et al., 2004; Liu y Wilson, 2002). En conclusión: las teorías y los estudios antes presentados señalan que tanto los factores internos como los externos son determinantes en la insolvencia empresarial.

El concepto de la insolvencia empresarial se define de dos formas cuando la empresa es incapaz de generar beneficios a largo plazo y cuando

Factores determinantes de la insolvencia empresarial: caso aplicado a la Bolsa Mexicana de Valores. 
se tiene la falta de liquidez para cumplir los compromisos en el corto plazo (Altman et al., 2019; Lizano y de Lema, 2010). Montalván et al. (2011) nombran a estas dos dimensiones de conceptos como insolvencia económica e insolvencia financiera respectivamente. Si ambas situaciones perduran en el tiempo la empresa se puede declarar en quiebra.

Para el caso de México existe la Ley de Quiebras y Suspensión de Pagos que define el término quiebra como un estado judicial que emite un juez cuando se presentan dos elementos jurídicos fundamentales: 1) la persona física o moral a quien se le va a declarar en quiebra sea comerciante y 2) dicha persona haya cesado pagos. Es necesario señalar que toda empresa que se declare en bancarrota es una empresa insolvente pero no toda empresa que sea insolvente tiene que estar en bancarrota (Diputados, 1990).

\section{Metodología}

El periodo de estudio de esta investigación fue de 1993 a 2018 seleccionando una muestra de 33 empresas que cotizan en la BMV, de las cuales 16 entraron en la suspensión de pagos y 17 permanecieron solventes y se escogieron de acuerdo con la similitud de las empresas tanto en tamaño como en el sector al que pertenecen. Se consultó la base de datos de Bloomberg para obtener las variables contables y de mercado. Para determinar el impacto de los factores internos y externos en la insolvencia empresarial en la muestra total optamos por estimar un modelo probit. El modelo probit parte del modelo lineal general (1):

$$
P_{i}=f\left(x_{i} \beta\right)+\mu_{i}
$$

Donde se tiene como variable dependiente una probabilidad se elige la función $f$ como una distribución de una Normal $(0,1)$ con la finalidad de que los valores de $P_{i}$ se encuentren siempre en el intervalo $[0,1]$ y se obtiene el modelo probit cuyos parámetros $\beta$ admiten estimación por máxima verosimilitud:

$$
f\left(x_{i t} \beta\right)=\int_{-\infty}^{x_{i t} \beta} \frac{1}{2 \pi} \exp \left(-\frac{1}{2} t^{2}\right) d t(2)
$$

\section{Estrada Berlanga et al.}


En el modelo probit al dotarse de estructura de datos panel se introduce un nuevo índice de variación temporal que puede escribirse como:

$$
\begin{gathered}
y_{i t}=(2 \pi)^{1 / 2} \int_{-\infty}^{x_{i t} \beta} \exp \left(-\frac{1}{2} p^{2}\right) d p+v_{i}=F\left(x_{i t} \beta+v_{i}\right) \\
t=1, \ldots, T ; \quad i=1, \ldots, N
\end{gathered}
$$

$y_{i t}$ es una variable latente igual a 1 si la empresa se encuentra en insolvencia y 0 en caso contrario, i representa las unidades de corte transversal que en nuestra investigación corresponden a las empresas y $t$ tiempo que se encuentra representado por años del período de 1993-2018. $x^{\prime}{ }_{i t}$ son los factores determinantes de la insolvencia empresarial, $\beta$ incluye los estimadores que se deben estimar. La estimación de los parámetros por máxima verosimilitud nos lleva a maximizar la función:

$$
g\left(x_{i t}, v_{i}\right)=\frac{e^{-v_{i}^{2} / 2 \sigma_{v}^{2}}}{\sqrt{2 \pi \sigma_{v}}}\left\{\prod F\left(x_{i t} \beta+v_{i}\right)\right\}
$$

donde $g$ es una distribución que obtiene la estimación por máxima verosimilitud de los parámetros del modelo probit (Pérez, 2006).

Los efectos marginales del método probit pueden interpretarse de dos formas. La primera como el efecto promedio en toda la muestra de un cambio de una unidad en las variables independientes de interés sobre la probabilidad de que presente insolvencia empresarial. La segunda como el cambio en la proporción de observaciones que presentan insolvencia debido a un cambio en las variables independientes (Méndez et al., 2019).

Briones et al. (1987) nos indican la importancia de utilizar un modelo probit en los métodos de predicción dado que realizaron un análisis comparativo de los modelos estadísticos predictivos en donde comparan el modelo lineal discriminante, técnica implementada por Altman (1968), con el modelo de variable binaria. Los porcentajes de clasificación correcta de un modelo de variable binaria resultan más elevados, además el porcentaje de aciertos en las empresas fracasadas es mucho mayor con el modelo de variable binaria que con el modelo discriminante, lo cual es altamente significativo teniendo en cuenta que el coste de clasificación errónea de las empresas fracasadas es mucho mayor.

\section{Factores determinantes de la insolvencia empresarial:} caso aplicado a la Bolsa Mexicana de Valores. 
Se puede concluir que la regresión probabilística es el método más recomendado para predecir la insolvencia empresarial (Cueto et al.,1985; Pereira et al., 2007; Kumar y Ravi, 2007; Aziz y Dar, 2006). En la presente investigación se pretende determinar si los factores internos y externos tienen efecto en la insolvencia empresarial en la BMV. Nuestras hipótesis planteadas para esta investigación son las siguientes:

H1: los factores financieros, no financieros y macroeconómicos impactan en la insolvencia empresarial.

H2: los sectores empresariales tienen diferente riesgo de caer en insolvencia empresarial en las empresas que cotizan en la BMV.

Para probar la hipótesis uno primero estimaremos la siguiente ecuación:

$$
\begin{gathered}
y_{i t}=I E_{i t}=\beta_{0}+\beta_{1} R O A_{i t}+\beta_{2} \text { Liq }_{i t}+\beta_{3} \text { End }_{i t}+\beta_{4} F E O_{i t} * F E+ \\
\beta_{5} \text { Tam }_{i t}+\beta_{6} \text { Edad }_{i t}+\beta_{7} \text { Crec }_{i t}+\beta_{8} \text { Embit }_{i t}+\beta_{10} \text { beta }_{i t}+ \\
\beta_{11} R E_{i t}+\beta_{12} T C N_{i t}+e_{i t}
\end{gathered}
$$

Donde:

$I E=\quad$ Es una variable que toma el valor de 1 cuando la empresa está en un estado de insolvencia empresarial y 0 en caso contrario para la empresa $i$ en el ejercicio $t$.

$R O A=$ Es el rendimiento sobre los activos calculado mediante la utilidad neta entre el promedio de los activos para la empresa $i$ en el ejercicio $t$.

Liq $=\quad$ Es la liquidez de la empresa medida por los activos corrientes entre los pasivos corrientes para la empresa $i$ en el ejercicio $t$.

End $=$ Es el endeudamiento de la empresa medido por los activos totales entre los pasivos totales para la empresa $i$ en el ejercicio $t$.

$F E O=$ Es la relación del flujo de efectivo operativo de la empresa medido por el flujo de efectivo operativo entre las utilidades operativas para la empresa $i$ en el ejercicio $t$.

$F E=\quad$ Es el flujo de efectivo neto de la empresa medido a través del logaritmo neperiano de la sumatoria del flujo de efectivo operativo,

\section{Estrada Berlanga et al.}


flujo de efectivo de inversión y el flujo de efectivo del financiamiento para la empresa $i$ en el ejercicio $t$.

Tam = Es el tamaño de la empresa medido a través del logaritmo neperiano de los activos totales de las empresas para la empresa $i$ en el ejercicio $t$.

$E d a d=$ Es la edad de la empresa medido a través del logaritmo neperiano de la edad para la empresa $i$ en el ejercicio $t$.

Crec $=$ Es el crecimiento de la empresa medido por el logaritmo neperiano de las ventas del año actual menos el logaritmo neperiano de las ventas del año anterior para la empresa $i$ en el ejercicio $t$.

$E M B I T+=$ Es el riesgo país medido por el riesgo país de México entre el riesgo país de Latinoamérica para la empresa $i$ en el ejercicio $t$.

beta $=$ Es el riesgo del mercado en México para la empresa $i$ en el ejercicio $t$.

$R E=\quad$ Es una variable que toma el valor de 1 cuando la empresa está en una recesión económica y 0 en caso contrario para la empresa $i$ en el ejercicio $t$.

$T C N=$ Es la variación del tipo de cambio que tiene como base la moneda de dólar y se calcula con el logaritmo neperiano del tipo del cambio del año actual menos el logaritmo neperiano del tipo de cambio del año anterior para la empresa $i$ en el ejercicio $t$.

Posteriormente probaremos nuestra hipótesis dos mediante la siguiente ecuación:

$$
\begin{gathered}
I E_{i t}=\beta_{0}+\beta_{1} R O A_{i t}+\beta_{2} L i q_{i t}+\beta_{3} E n d_{i t}+\beta_{4} F E O_{i t}+\beta_{5} I N D_{i t}+ \\
\beta_{6} S B C N B_{i t}+\beta_{7} P C F_{i t}+\beta_{8} M A T_{i t}+e_{i t}
\end{gathered}
$$

Donde:

$I N D=\quad$ Variable dummie que toma valores de 1 si la empresa pertenece al sector industrial y 0 en caso contrario para la empresa $i$ en el ejercicio $t$.

$S B C N B=$ Variable dummie que toma valores de 1 si la empresa pertenece al sector de servicios y bienes de consumo no básico y 0 en caso contrario para la empresa $i$ en el ejercicio $t$.

Factores determinantes de la insolvencia empresarial: caso aplicado a la Bolsa Mexicana de Valores. 
$P C F=\quad$ Variable dummie que toma valores de 1 si la empresa pertenece al sector de productos de consumo frecuente y 0 en caso contrario para la empresa $i$ en el ejercicio $t$.

$M A T=$ Variable dummie que toma valores de 1 si la empresa pertenece al sector de materiales y 0 en caso contrario para la empresa $i$ en el ejercicio $t$.

\section{Resultados}

En la Tabla 1 se presentan las estadísticas descriptivas de las variables de los factores internos y los factores externos utilizadas en la ecuación 5 y 6 . En promedio los factores internos muestran que las empresas tienen $3.51 \%$ de rentabilidad sobre los activos, una liquidez de 2.48 representando un poco más del doble de los activos corrientes sobre los pasivos corrientes, un endeudamiento de 0.55 , esto representa más del doble de los activos totales sobre los pasivos totales, un flujo de efectivo operativo de 0.42 significa que el efectivo que se ha utilizado para la operación es la mitad de lo que se ha generado para las utilidades operativas y el total de flujo de efectivo neto son alrededor de los 4 millones por empresa. El tamaño de la empresa es de 9.56 que equivale a un poco más de 13 millones en activos totales, la edad tiene un indicador de 3.11 que equivale a un promedio de edad de 21 años y un crecimiento promedio en ventas de $4.23 \%$ año con año.

Los factores externos muestran un riesgo país de 0.40 siendo México un país menos riesgoso que el resto de Latinoamérica, un riesgo de mercado beta de 0.71 , lo que muestra que los activos de las empresas estudiadas tienen menor riesgo sistémico que el mercado es menos volátil que la tendencia general, una recesión de 0.40 que equivale a un poco menos de la mitad de situaciones de recesiones en México y el tipo de cambio neto casi cero que significa que es muy sensible a los cambios que tiene el comportamiento del dólar.

El sector industrial es el que menos participación tiene en la presente investigación con un $9.28 \%$, el sector de servicio y bienes de consumo no básico es el que mayor participación tiene con un $47.31 \%$ y finalmente los sectores de productos de consumo frecuente y el sector de materiales tuvieron la misma participación con un $24.79 \%$ respectivamente.

\section{Estrada Berlanga et al.}


Tabla 1. Estadísticas descriptivas.

\begin{tabular}{|c|c|c|}
\hline Variable & Media & Desviación Estándar \\
\hline${ }^{1} \mathrm{ROA}_{\mathrm{t}}$ & 0.035181 & 0.130711 \\
\hline${ }^{2}$ Liq $t$ & 2.487775 & 3.306651 \\
\hline${ }^{3}$ End $t$ & 0.558112 & 0.450817 \\
\hline${ }^{4} \mathrm{FEO} \mathrm{t}_{\mathrm{t}}$ & 0.429604 & 8.505041 \\
\hline${ }^{5} \mathrm{FE}_{\mathrm{t}}$ & 1.869804 & 35.00942 \\
\hline${ }^{6} \mathrm{Tam}_{\mathrm{t}}$ & 9.562577 & 1.326497 \\
\hline${ }^{7} \mathrm{Edad}_{\mathrm{t}}$ & 3.117578 & 0.754042 \\
\hline${ }^{8} \mathrm{Crec} t$ & 0.042384 & 0.351936 \\
\hline${ }^{9} \mathrm{EMBIT}+\mathrm{t}$ & 0.400666 & 0.273840 \\
\hline${ }^{10}$ beta $\mathrm{t}$ & 0.717478 & 0.349942 \\
\hline${ }^{11} \mathrm{RE}_{\mathrm{t}}$ & 0.403880 & 0.491107 \\
\hline${ }^{12} \mathrm{TCN}_{\mathrm{t}}$ & 0.001463 & 0.123224 \\
\hline${ }^{13} \mid N D_{t}$ & 0.092968 & 0.290561 \\
\hline${ }^{14} \mathrm{SBCNB}_{\mathrm{t}}$ & 0.473182 & 0.499578 \\
\hline${ }^{15} \mathrm{PCF}_{\mathrm{t}}$ & 0.247914 & 0.432059 \\
\hline${ }^{16} \mathrm{MAT}_{\mathrm{t}}$ & 0.247914 & 0.610600 \\
\hline
\end{tabular}

Fuente: Elaboración propia usando E-views 7 basada en Bloomberg.

Debido a que la variable dependiente es dicotómica la varianza del modelo es heterocedástica y los datos muestran no normalidad. Al tener estos comportamientos se aplica un corrector Huber/White con la finalidad de corregir las covarianzas que presentan los datos y solucionar el problema (Startz, 2012).

En la Tabla 2 se presentan los resultados del primer modelo financiero y económico. De acuerdo con los resultados el modelo presenta $96.86 \%$ de asertividad y un indicador de McFadden R-cuadrada del $82.08 \%$.

Tabla 2. Resultados del modelo financiero y económico.

\begin{tabular}{lcccc}
\hline \multicolumn{1}{c}{ Variable } & Coeficiente & Error Estándar & Estadístico Z & Probabilidad \\
\hline${ }^{\mathrm{C}}$ & & & & \\
${ }^{1} \mathrm{ROA}_{\mathrm{i}, \mathrm{t}}$ & -6.812458 & 2.136181 & -3.189083 & 0.0014 \\
${ }^{2}{ }^{\mathrm{Liq}} \mathrm{i}, \mathrm{t}$ & -4.376904 & 1.496027 & -2.925685 & 0.0034 \\
${ }^{3}{ }^{\mathrm{End}} \mathrm{i}, \mathrm{t}$ & -4.338961 & 1.050220 & -4.131478 & 0.0000 \\
${ }^{4} \mathrm{FE}_{\mathrm{i}, \mathrm{t}-\mathrm{t}}$ & 4.862233 & 1.449733 & 3.353880 & 0.0008 \\
${ }^{5} \mathrm{Tam}_{\mathrm{i}, \mathrm{t}}$ & -0.035774 & 0.021032 & -1.700967 & 0.0889 \\
${ }^{6} \mathrm{Edad}^{2}{ }_{\mathrm{i}, \mathrm{t}}$ & 0.463144 & 0.192417 & 2.406977 & 0.0161 \\
${ }^{7}{ }^{\mathrm{EMBIT}}{ }^{\text {beta }}{ }_{\mathrm{i}, \mathrm{t}}$ & 0.132026 & 0.050628 & 2.607738 & 0.0091 \\
${ }^{8} \mathrm{RE}_{\mathrm{i}, \mathrm{t}}$ & -3.421577 & 1.115142 & -3.068287 & 0.0022 \\
${ }^{9}{ }^{\mathrm{TCN}} \mathrm{t}-3$ & -1.346519 & 0.532622 & -2.528096 & 0.0115 \\
\end{tabular}

Fuente: Elaboración propia usando E-views 7 basada en Bloomberg.

Factores determinantes de la insolvencia empresarial: caso aplicado a la Bolsa Mexicana de Valores. 
Los factores internos muestran que las variables de ROA $\left(\beta_{1}=-4.376904, p<\right.$ $0.0034)$ y Liq $\left(\beta_{2}=-4.338961, p<0.0001\right)$ impactan con signo negativo y son significativas sobre la insolvencia empresarial que indica a mayor cantidad de estas razones financieras es menos probable que una empresa pueda caer en una insolvencia empresarial. Caso contrario fueron las variables de End $\left(\beta_{3}=\right.$ 4.862233, $p<0.0008), \operatorname{Tam}\left(\beta_{5}=0.463144, p<0.0161\right)$ y $\operatorname{Edad}^{2}\left(\beta_{6}=\right.$ $0.132026, p<0.0091)$ muestran un signo positivo y son significativas sobre la insolvencia empresarial, lo cual nos dice que a mayor cantidad sobre estos indicadores es más probable que una empresa pueda estar en un estado de insolvencia empresarial. Por último la variable de FE $\left(\beta_{4}=-0.035774, p<\right.$ 0.0889 ) indica un comportamiento contrario a la insolvencia pero no fue significativo.

Los factores externos muestran que las variables de EMBIT*beta $\left(\beta_{7}=\right.$ $-3.421577, p<0.0022)$, y $\operatorname{RE}\left(\beta_{8}=-1.346519, p<0.0115\right)$ tuvieron un signo negativo y fueron significativas esto muestra que un mejor indicador de la variable EMBIT multiplicada por beta y de RE disminuye la probabilidad de caer en una insolvencia empresarial. La variable TCN $\left(\beta_{9}=10.34044, p<\right.$ $0.0069)$ con tres años de anterioridad está relacionada de manera positiva y es significativa con la posibilidad de caer en una insolvencia empresarial. En la Tabla 3 se muestran los resultados de los efectos marginales para los coeficientes del modelo financiero-económico.

Tabla 3. Efectos marginales modelo financiero-económico.

\begin{tabular}{|c|c|c|}
\hline Variable & Coeficiente & Probabilidad \\
\hline${ }^{1} \mathrm{ROA} \mathrm{A}_{\mathrm{i}, \mathrm{t}}$ & -1.7461 & 0.0034 \\
\hline${ }^{2} \mathrm{Liq} i, \mathrm{t}$ & -1.7310 & 0.0000 \\
\hline${ }^{3}$ End $_{\mathrm{i}, \mathrm{t}}$ & 1.9398 & 0.0008 \\
\hline${ }^{4} \mathrm{FE}_{\mathrm{i}, \mathrm{t}-\mathrm{1}}$ & -0.0143 & 0.0889 \\
\hline${ }^{5} \operatorname{Tam}_{i, t}$ & 0.1848 & 0.0161 \\
\hline${ }^{6} \mathrm{Edad}^{2} \mathrm{i}, \mathrm{t}$ & 0.0527 & 0.0091 \\
\hline${ }^{7} \mathrm{EMBIT}^{*}$ beta $_{\mathrm{i}, \mathrm{t}}$ & -1.3650 & 0.0022 \\
\hline${ }^{8} \mathrm{RE}_{\mathrm{i}, \mathrm{t}}$ & -0.5372 & 0.0115 \\
\hline${ }^{9} \mathrm{TCN}{ }_{\mathrm{t}-3}$ & 4.1252 & 0.0069 \\
\hline
\end{tabular}

Fuente: Elaboración propia usando E-views 7 basada en Bloomberg.

En relación con los factores internos un incremento de $1 \%$ en la variable ROA aumentará la probabilidad de no caer en insolvencia en un $174 \%$, de la misma

\section{Estrada Berlanga et al.}


forma un incremento de $1 \%$ en la variable Liq aumentará la probabilidad de no caer en insolvencia en un $173 \%$. Caso contrario con el comportamiento de la variable de End un incremento del 1\% aumentará la probabilidad en un 193\% de estar en insolvencia empresarial, un incremento del $1 \%$ en la variable Tam aumentará un $18 \%$ la probabilidad de caer en insolvencia, un incremento de $1 \%$ en la variable Edad 2 subirá un $5 \%$ la probabilidad de caer en insolvencia empresarial.

Los factores externos nos muestran un alza del $1 \%$ en las variables EMBIT*beta incrementará en un $136 \%$ la probabilidad de no caer en insolvencia empresarial y mismo caso es la variable RE: un incremento del $1 \%$ aumentará $5 \%$ la probabilidad de no caer en insolvencia empresarial. De forma contraria un incremento del $1 \%$ en la variable TCN aumentará la probabilidad de estar en un estado de insolvencia empresarial en un $412 \%$.

Los resultados del modelo multisectorial se muestran en la Tabla 4. De acuerdo con los resultados el modelo presenta $94.36 \%$ de asertividad y un indicador de McFadden R-cuadrada del $62.55 \%$.

Tabla 4. Resultado del modelo multisectorial.

\begin{tabular}{|c|c|c|c|c|}
\hline Variable & Coeficiente & $\begin{array}{c}\text { Error } \\
\text { Estándar }\end{array}$ & Estadístico Z & Probabilidad \\
\hline$C$ & 0.392469 & 0.527986 & 0.743332 & 0.4573 \\
\hline${ }^{1} \mathrm{ROA}_{\mathrm{i}, \mathrm{t}}$ & -1.914148 & 0.846703 & -2.260708 & 0.0238 \\
\hline${ }^{2} \mathrm{Liq}_{\mathrm{i}, \mathrm{t}}$ & -2.313950 & 0.374959 & -6.171212 & 0.0000 \\
\hline${ }^{3} E_{n d}, t$, & 1.530548 & 0.453285 & 3.376570 & 0.0007 \\
\hline${ }^{4} \mathrm{FEO}_{\mathrm{i}, \mathrm{t}}$ & -0.004338 & 0.006887 & -0.629970 & 0.5287 \\
\hline${ }^{13} \mid \mathrm{ND} \mathrm{i}, \mathrm{t}$ & -1.305425 & 0.318855 & -4.094102 & 0.0000 \\
\hline${ }^{14} \mathrm{SBCNB} \mathrm{i}_{\mathrm{i}, \mathrm{t}}$ & -1.351940 & 0.383651 & -3.523881 & 0.0004 \\
\hline${ }^{15} \mathrm{PCF}_{\mathrm{i}, \mathrm{t}}$ & -0.711015 & 0.333252 & -2.133566 & 0.0329 \\
\hline
\end{tabular}

McFadden Rsquared 0.6255.

Porcentajes $\quad 94.36$.

correctos $\%$.

Fuente: Elaboración propia usando E-views 7 basada en Bloomberg.

El efecto de las variables de ROA, Liq y End presentan el mismo comportamiento de los resultados del modelo financiero-económico. La variable FEO no resultó significativa en este modelo. Los resultados muestran que el sector de los productos de consumo frecuente son el de mayor impacto con un signo positivo que indica que este sector es el de mayor riesgo de caer en insolvencia con respecto al resto de los sectores seguido por el sector

Factores determinantes de la insolvencia empresarial: caso aplicado a la Bolsa Mexicana de Valores. 
industrial con un mejor grado de significancia y por último el sector de servicios y bienes de consumo no básico, todos con un signo negativo y significativo que impacta en la probabilidad de tener insolvencia empresarial.

\section{Conclusiones}

En el presente artículo se estimaron dos modelos utilizando el método probit: en el primer modelo se analizó el impacto de los factores financieros, no financieros y macroeconómicos en la insolvencia empresarial de las empresas que cotizan en la BMV y en el segundo modelo se analizaron los sectores que mayor riesgo tienen de caer en insolvencia con respecto al resto de los sectores de las empresas que cotizan en la BMV. Se utilizaron datos de Bloomberg para obtener las variables contables y de mercado. $Y$ de los resultados obtenidos del presente artículo se generan tres principales conclusiones:

1) Conforme aumenten el nivel de endeudamiento, tamaño, edad y el tipo de cambio en referencia al dólar en México se genera una mayor probabilidad de caer en una insolvencia empresarial en las empresas públicas que participan en la BMV. Caso contrario si la rentabilidad, la liquidez, el flujo de efectivo neto, el flujo de efectivo operativo, el riesgo de país, la beta de los mercados y las recesiones económicas crecen es menos probable que una empresa pueda entrar en un estado de insolvencia empresarial. Esto concuerda con los estudios de Almamy et al. (2016), Arroyave, (2018), Beaver et al. (2005), Darrat et al. (2016), Espinosa et al. (2015), Tinoco y Wilson, 2013, Abidali y Harris, (1995), Hsu y Wu, (2014), Platt y Platt, (2012) y MadridGuijarro et al. (2011) donde indican que las variables de mayor impacto en un modelo de insolvencia empresarial son los factores financieros y los no financieros. Li y Liu (2009) y Montalván et al. (2011) demostraron que agregar a los modelos de predicción variables macroeconómicas, éstas aumentaban el nivel de significancia y lo cual es similar a los resultados que obtuvo la presente investigación.

2) Los sectores con mayor probabilidad de caer en insolvencia empresarial fue el sector de producto de consumo básico, sector industrial y sector de servicios y bienes de consumo no básico mostrando un signo negativo y significativo en referencia a otros sectores. El sector de materiales es el que menos probabilidad tuvo de caer en insolvencia con respecto al resto de los sectores. Estos resultados concuerdan con dos estudios: el de

\section{Estrada Berlanga et al.}


Rodríguez et al. (2015) y el de Mascareñas y Alvarez-Pallete (2006). Rodríguez et al. (2015) indican que los sectores con mejores niveles de desempeño financiero son el sector de materiales y uno de los sectores que menos desempeño presenta en México es el sector industrial. Mascareñas y Alvarez-Pallete (2006) mencionan que el aumento de la demanda que China ha tenido por las materias primas en Latinoamérica ha beneficiado al sector de materiales. El sector industrial está compuesto principalmente por empresas de construcción, giro que ha sido golpeado mucho en los últimos años (Yarza, 2019).

3) Los modelos de predicción que generó la presente investigación pueden ayudar a la prevención y redirección del rumbo de la empresa en busca de generar más y mayores beneficios. Los modelos de predicción históricamente han ayudado a entidades dedicadas al análisis del riesgo crediticio, bancos, inversionistas, gobiernos e instituciones internacionales a tomar acciones anticipadas que beneficien a la sociedad para la generación del empleo y el bienestar social, como Altman et al. (2019) han utilizado su modelo de Z-score, y a su vez los resultados de esta investigación aportan al desarrollo de la teoría de la insolvencia empresarial ofreciendo un mejor entendimiento de sus determinantes para que eventualmente se pueda sumar a los esfuerzos científicos en la construcción de un marco teórico que explique la quiebra de las empresas. La presente investigación se encontró limitada por la falta de registro de la información de todas las empresas que han presentado insolvencia empresarial. Sin embargo, se consideraron sólo una parte de las empresas que han caído en este estado, además no se consideraron los perfiles de los tomadores de decisiones. Actualmente las tecnologías financieras tienen un papel importante en el desempeño de las empresas. Aún no se cuenta con los datos necesarios para poder hacer algún análisis sobre éste.

Los retos para futuras investigaciones serían incorporar el uso de las tecnologías financieras y los perfiles de los tomadores de decisiones de las empresas al análisis de la insolvencia empresarial. Además, se podría crear un modelo basado en la metodología Kaplan-Meier para calcular el tiempo estimado de vida para cada empresa.

Y por último se cumplió el objetivo del estudio y se obtuvieron resultados contundentes demostrando que los factores financieros, no financieros y macroeconómicos son los determinantes en la insolvencia

Factores determinantes de la insolvencia empresarial: caso aplicado a la Bolsa Mexicana de Valores. 
empresarial en México. Además, se determinaron cuáles son los sectores más sensibles de caer en una insolvencia empresarial.

\section{Estrada Berlanga et al.}




\section{Referencias}

Abidali, A. F., y Harris, F. (1995). A methodology for predicting company failure in the construction industry. Construction management and economics, 13(3), 189-196.

Agarwal, V. y Taffler, R. (2008). Comparing the performance of market-based and accounting-based bankruptcy prediction models. Journal of Banking \& Finance, 32(8), 1541-1551.

Almamy, J., Aston, J., y Ngwa, L. N. (2016). An evaluation of Altman's Z-score using cash flow ratio to predict corporate failure amid the recent financial crisis: Evidence from the UK. Journal of Corporate Finance, 36, 278-285.

Altman, E. I. (1968). Financial ratios, discriminant analysis and the prediction of corporate bankruptcy. The journal of finance, 23(4), 589-609.

Altman, E. I. y Suggitt, H. J. (2000). Default rates in the syndicated bank loan market: A mortality analysis. Journal of Banking \& Finance, 24(1-2), 229-253.

Altman, E., Hartzell, J., Peck, M., Levich, R. y Mei, J. (1995). Future of emerging market flows. New York: Salomon Brothers, Inc.

Arroyave, J. (2018). A comparative analysis of the effectiveness of corporate bankruptcy prediction models based on financial ratios: Evidence from Colombia. Journal of International Studies, 11(1), 273-287.

Ayús, A. L. T., Velásquez, R. E. A. y Ceballos, H. V. (2010). Estimación de las provisiones esperadas en una institución financiera utilizando modelos Logit y Probit. Revista Ciencias Estratégicas, 18(24), 259-270.

Aziz, A. y Lawson, G. H. (1989). Cash flow reporting and financial distress models: Testing of hypotheses. Financial Management, 55-63.

Aziz, M. A. y Dar, H. A. (2006). Predicting corporate bankruptcy: where we stand? Corporate Governance: The international journal of business in society.

Bathory, A. (1984). Predicting Corporate Collapse: Credit Analysis in the Determination and Forecasting of Insolvent Companies. Financial Times Business Information.

Beaver, W. H. (1966). Financial ratios as predictors of failure. Journal of accounting research, 71-111.

Factores determinantes de la insolvencia empresarial: caso aplicado a la Bolsa Mexicana de Valores. 
Beaver, W. H., McNichols, M. F. y Rhie, J. W. (2005). Have financial statements become less informative? Evidence from the ability of financial ratios to predict bankruptcy. Review of Accounting studies, 10(1), 93-122.

BM. (04 de Junio de 2019). Global growth to weaken to 26 in 2019 substantial risks seen. Obtenido de Banco Mundial:

https://www.bancomundial.org/es/news/pressrelease/2019/06/04/global-growth-to-weaken-to-26-in-2019substantial-risks-seen

Brealey, R. A., Myers, S. C., Allen, F., Soria, L. N. y Izquierdo, M. Á. F. (2015). Principios de finanzas corporativas. McGraw-Hill Interamericana.

Briones, J. L., Marín, J. L. M. y Cueto, M. J. V. (1987). Predicción de la crisis bancaria en España: comparación entre el análisis logit y el análisis discriminante. Cuadernos de Ciencias Económicas y Empresariales, (18), 49-57.

Bukovinsky, D. M. (1994). Cash flow and cash position measures in the prediction of business failure: An empirical study.

Cabrera, A. A., Ayala, Á. A., Perus, M. C., M, S. Y., Ortega, A. H. y Peschard, J. (2006). Efectos psicosociales del desempleo. Revista de Investigación Social, UNAM, 67-82.

Campbell, J. Y., Hilscher, J. D. y Szilagyi, J. (2011). Predicting financial distress and the performance of distressed stocks. Journal of Investment Management.

Casey, C. y Bartczak, N. (1985). Using operating cash flow data to predict financial distress: Some extensions. Journal of Accounting Research, 384-401.

Christidis, A. y Gregory, A. (2010). Some new models for financial distress prediction in the UK. Xfi-Centre for Finance and Investment Discussion Paper, (10).

Cueto, M. J. V., Marín, J. L. M. y Briones, J. L. (1985). El análisis de la solvencia en las instituciones bancarias: Propuesta de una metodología y aplicaciones a la Banca española. Esic Market, (48), 51-73.

Dambolena, I. G. y Khoury, S. J. (1980). Ratio stability and corporate failure. The Journal of Finance, 35(4), 1017-1026.

\section{Estrada Berlanga et al.}


Darrat, A. F., Gray, S., Park, J. C. y Wu, Y. (2016). Corporate governance and bankruptcy risk. Journal of Accounting, Auditing \& Finance, 31(2), 163202.

Diputados, C. d. (1990). Ley de quiebras y suspensión de pagos. http://www.diputados.gob.mx/bibliot/publica/inveyana/polint/dpi05/1m arco.htm

Espinosa, F. R., Molina, Z. A. M. y Vera-Colina, M. A. (2015). Fracaso empresarial de las pequeñas y medianas empresas (pymes) en Colombia. Suma de negocios, 6(13), 29-41.

Fitzpatrick, P. J. (1932). A comparison of the ratios of successful industrial enterprises with those of failed companies.

Forrester, J. W. (1968). Industrial dynamics-after the first decade. Management Science, 14(7), 398-415.

Foster, G. Financial Statement Analysis, 1986.

Freire, A. G. H., Gonzaga, V. A. B., Freire, A. H. H., Rodríguez, S. R. V. y Granda, E. C. V. (2016). Razones financieras de liquidez en la gestión empresarial para toma de decisiones. Quipukamayoc, 24, 46.

García, M. D. P. R., Alejandro, K. A. C., Sáenz, A. B. M. y Sánchez, H. H. G. (2015). Análisis de portafolio por sectores mediante el uso de algoritmos genéticos: caso aplicado a la Bolsa Mexicana de Valores. Contaduría y administración, 60(1), 87-112.

García, M. L., Vega, T. d. y Uribe, H. M. (2009). Impacto social de una quiebra: Caso de la empresa automotriz mexicana Dina S.A. Economía XXXIV, $28,75-101$.

Gentry, J. A., Newbold, P. y Whitford, D. T. (1985). Predicting bankruptcy: if cash flow's not the bottom line, what is? Financial Analysts Journal, 41(5), 47-56.

Gombola, M. J., Haskins, M. E., Ketz, J. E. y Williams, D. D. (1987). Cash flow in bankruptcy prediction. Financial Management, 55-65.

Hillegeist, S. A., Keating, E. K., Cram, D. P. y Lundstedt, K. G. (2004). Assessing the probability of bankruptcy. Review of accounting studies, 9(1), 5-34.

Hsu, H. H. y Wu, C. Y. H. (2014). Board composition, grey directors and corporate failure in the UK. The British Accounting Review, 46(3), 215227.

Factores determinantes de la insolvencia empresarial: caso aplicado a la Bolsa Mexicana de Valores. 
Ibarra Mares, A. (2002). Análisis de las dificultades financieras de las empresas en una economía emergente las bases de datos y las variables independientes en el sector hotelero de la bolsa mexicana de valores. Universitat Autònoma de Barcelona.

INEGI. (05 de Enero de 2016). Instituto Nacional de Estadística y Geografía. Obtenido de Esperanza de vida de los negocios a nivel nacional y por entidad federativa. https://www.inegi.org.mx/temas/evnm/default.html\#Informacion_gene ral

Katz, D. y Kahn, R. L. (1978). Organizations and the system concept. Classics of organization theory, $80,480$.

Kim, S., Mun, B. M. y Bae, S. J. (2018). Data depth-based support vector machines for predicting corporate bankruptcy. Applied Intelligence, 48(3), 791-804.

Kuger, M. (2019). Global Bankruptcy Report 2019. Nueva Jersey, EEUU: Dun \& Bradstreet Worldwide Network.

Kumar, P. R. y Ravi, V. (2007). Bankruptcy prediction in banks and firms via statistical and intelligent techniques-A review. European journal of operational research, 180(1), 1-28.

Lennox, C. (1999). Identifying failing companies: a re-evaluation of the logit, probit and DA approaches. Journal of economics and Business, 51(4), 347-364.

Li, D. y Liu, J. (2009). Determinants of financial distress of ST and PT companies: A panel analysis of chinese listed companies. Disponible en el SSRN: http://ssrn.com/abstract=1341795.

Liu, J. y Wilson, N. (2002). Corporate failure rates and the impact of the 1986 insolvency act: An econometric analysis. Managerial Finance, 28(6), 61-71.

Lizano, M. M., Ochovo, R. B. y de Lema, D. G. P. (2010). Diferentes procesos de fracaso empresarial: un análisis dinámico a través de la aplicación de técnicas estadísticas clúster. Revista Europea de Dirección y Economía de la Empresa, 19(3), 67-88.

Madrid-Guijarro, A., García-Pérez-de-Lema, D. y Van Auken, H. (2011). An analysis of non-financial factors associated with financial distress. Entrepreneurship and Regional Development, 23(3-4), 159186.

\section{Estrada Berlanga et al.}


Mascareñas, J. y Alvarez-Pallete, J. M. (2006). China-Latinoamérica: ¿competencia o cooperación? Documento de Trabajo, 504.

Méndez Sáenz, A. B., Rodríguez García, M. D. P. y Cortez Alejandro, K. A. (2019). Factores Determinantes de la Responsabilidad Social Empresarial (RSE). Caso aplicado a México y Brasil. Análisis económico, 34(86), 197-217.

Montalván, S. M., Delgado, F. I. A., O'Shee, D. F. y Yamashiro, M. A. (2011). Determinantes de la insolvencia empresarial en el Perú. Academia. Revista latinoamericana de administración, (47), 126-139.

Ohlson, J. A. (1980). Financial ratios and the probabilistic prediction of bankruptcy. Journal of accounting research, 109-131.

Pereira, J. M., Domínguez, M. Á. C. y Ocejo, J. L. S. (2007). Modelos de previsão do fracasso empresarial: aspectos a considerar. TékhneRevista de Estudos Politécnicos, (7), 111-148.

Pérez López, Cesar (2006). Problemas resueltos de econometría. Editorial Paraninfo.

Pervan, I. y Kuvek, T. (2013). The relative importance of financial ratios and nonfinancial variables in predicting of insolvency. Croatian Operational research review, 4(1), 187-197.

Pinzón, O. L. (2017). Causas e impacto socio-económico por liquidación de las empresas comerciales y de servicios. FACE Volumen 17- $N^{\circ} 1,44-54$.

Platt, H. y Platt, M. (2012). Corporate board attributes and bankruptcy. Journal of Business Research, 65(8), 1139-1143.

Rahimian, E., Singh, S., Thammachote, T. y Virmani, R. (1993). Bankruptcy prediction by neural network. Neural networks in finance and investing, 159-176.

Rees, B. (1990). Financial analysis. Londres: Prentice Hall.

Ruiz Palomo, D. (2010). La solvencia empresarial: pirámides de ratios vs. marco teórico.

Schmalensee, R. (1985). Do markets differ much? The American economic review, 75(3), 341-351.

Segura, A. S. (1994). La rentabilidad económica y financiera de la gran empresa española. Análisis de los factores determinantes. Revista española de financiación y contabilidad, 159-179.

Simon, H. A. (1962). La toma de decisiones y la organización administrativa. El comportamiento administrativo. Madrid: Aguilar.

\section{Factores determinantes de la insolvencia empresarial:} caso aplicado a la Bolsa Mexicana de Valores. 
Startz, R. (2012). Bayesian Heteroskedasticity-Robust Standard Errors.

Tinoco, M. H. y Wilson, N. (2013). Financial distress and bankruptcy prediction among listed companies using accounting, market and macroeconomic variables. International Review of Financial Analysis, 30, 394-419.

Uhrig-Homburg, M. (2005). Cash-flow shortage as an endogenous bankruptcy reason. Journal of Banking \& Finance, 29(6), 1509-1534.

Winakor, A. y Smith, R. (1935). Changes in the financial structure of unsuccessful industrial corporations. Bulletin, 51, 44.

Winter, S. G. (1996). Coase, la competencia y la corporación. Oliver E. Williamson y Sidney G. Winter (comps.), La naturaleza de la empresa. Orígenes, evolución y desarrollo. México: FCE.

Yarza, J. (19 de Agosto de 2019). Deloitte. Desaceleración en la construcción ¿Coyuntura o caída en picada?: https://www2.deloitte.com/mx/es/pages/dnoticias/articles/desacelerac ion-en-la-construccion.html\#

Zmijewski, M. E. (1984). Methodological issues related to the estimation of financial distress prediction models. Journal of Accounting research, 59-82.

\section{Estrada Berlanga et al.}

